$\left(25^{\circ}-32^{\circ} \mathrm{C}\right.$.) or relative humidity $(64 \%-84 \%)$. Because of the close correlation between these two variables, it was not possible to separate their effects on the eclampsia rate. Direct account could not be taken of domiciliary confinements in the city, though there was reason to believe that all eclamptic women were admitted to hospital.

It could be argued, perhaps, that cool or humid days might persuade some normal women to stay at home to deliver while those with eclampsia would continue to enter hospital-thus creating the impression in hospital that the eclampsia rate was higher on such days. However, this effect of coolness and humidity was considered to be real, since even a mild degree of coolness had an appreciable effect on the eclampsia rate, whereas rainfall had none. The absence of any significant correlation between humidity or temperature and blood pressure or oedema suggests that the effect was not mediated by vasospasm or fluid retention. Moreover the effect of cool weather was too large to be explained by a reduction in sweating, with a consequent increased likelihood of eclampsia through persistence of fluid retention. The nature of the connexion between eclampsia and the weather therefore remains obscure.

The immediate public health implications of the findings are minimal, but clearly it would be of interest to see if the relationship can be confirmed in other parts of the world. Meanwhile, it seems that eclampsia may have to be added to that varied group of conditions which are affected by the weather: which in addition to respiratory infections, includes ischaemic heart disease, peripheral vascular disease, systemic lupus erythematosus, and arthralgia-not to mention emotional states.

1 Neutra, R., Fournal of Obstetrics and Gynaecology of the British Commonwealth, 1974, 81, 833.

\section{Management of Recurrent Head and Neck Cancer}

The reappearance of a neoplasm following potentially curative therapy by radiation or surgery is conventionally described as a "recurrence," whether it is residual tumour left after ineffective resection, regional metastasis, or regrowth at the initial site after radiotherapy. Some tumour areas in the head and neck carry a markedly better prognosis than others, so overall recurrence rate will vary with the total experience of the surgeon as well as the distribution of the tumours he is treating. Despite the failure of the initial treatment, some patients can still be cured of their neoplasms; others can be offered only palliation of varying duration and effectiveness. The choice of this second line therapy and its successful accomplishment require considerable experience and adequate supporting facilities if the patient's sufferings are not to be increased. This must of necessity mean a degree of specialization, for such knowledge can be gained only by the care of large numbers of patients.

Inadequate surgical excision of the original tumour can rarely be rectified by a second operation. Radiotherapy has little to offer in the enhancement of cure rates in these patients but may have some palliative value, particularly the increasingly popular fast-neutron therapy.

Williams ${ }^{1}$ has recently emphasized the varying salvage rates for recurrent tumours in differing sites of the head and neck, and though his personal series was small-144 patients treated in six years (about the number seen by most large centres in less than a year)-his conclusions were apt. Tumours arising in areas inaccessible to radical excision are rarely cured if they recur after initial therapy-usually radiotherapy alone or in combination with an attempted radical excision. Nasopharyngeal carcinoma and tumours of the middle ear, base of tongue, and paranasal sinus are examples of this problem. On the other hand, secondary total laryngectomy or the technically more demanding pharyngolaryngoesophagectomy yields a generous proportion of long-term cures if carried out adequately and early in the recurrence-it is sometimes forgotten that there are degrees of recurrence and technical limitations to even the most enthusiastic surgeon's expertise. Extension to the skin and the need for its replacement require knowledge of the proper techniques of reconstruction, for there are inherent difficulties in the application of standard plastic procedures to previously irradiated tissues.

The problems in carrying out salvage procedures for tumours recurring in the oral cavity, where radiotherapy is invariably used as the initial mode of treatment, have been discussed recently by Harrison. ${ }^{2}$ Adequate excision is often possible, though it is of limited value if unaccompanied by effective rehabilitation of swallowing. Total glossectomy is a formidable procedure, even for young patients, while replacement of the whole or part of the mandible may be impossible after radiotherapy and suggests a need for urgent reappraisal of the initial therapy in these tumours.

Recurrence rates always reflect not only the effectiveness of the initial treatment but the incidence at sites, such as upper jaw and middle ear, which carry a very poor prognosis. Undoubtedly a combination of well-planned and carefully executed salvage surgery will result in the cure of some patients with recurrent disease arising at an advantageous site. Invariably the price to be paid will be high, not merely in money but, more important, in time and hardship. In the frail and the elderly this price may be too much. Perhaps a more rewarding approach would be better planned and executed primary treatment.

1 Williams, R. G., British fournal of Surgery, 1974, 61, 691.

${ }^{2}$ Harrison, D. F. N., Proceedings of the Royal Society of Medicine, 1974, 671 601 .

\section{Open Minds for Open Medicine}

Campaigning is under way for the Open University to start a medical faculty, in which mature students would pursue a part-time course at their own pace for some years and then, after passing examinations, become full time for their clinical training. Their medical qualifications would be no different from those granted by the existing medical schools in Britain.

On the face of it this seems an attractive proposition-a welcome supply of home-grown, dedicated doctors, often with previously learned skills, perhaps more interested in care than cure, produced at little expense; frustrated citizens turned, after character-proving struggle, to fulfilment; and a new use for the Open University's technical virtuosity. These were some of the benefits claimed at a conference on "Open Medicine and its Implications" held last weekend in Liverpool and organized by the Open Medicine Trust. ${ }^{1}$

Many people at the conference clearly thought that an Open University medical faculty was technically possible; but there 